\title{
Robust Non-Overshoot Time Responses using Cascade Sliding Mode-PID Control
}

\author{
T.H. Tran, Q.P. Ha, and H.T. Nguyen \\ Faculty of Engineering, University of Technology, Sydney \\ PO Box 123, Broadway NSW 2007, Australia \\ E-mail: $\{$ ttran|quangha|htn\}@eng.uts.edu.au
}

\begin{abstract}
Overshoot is a serio us problem in automatic control systems. This paper presents a new method for elimination of the step response overshoot in a conventional PID-controlled system and enhancement of its robustness by cascading a sliding mode controller in the outer loop. The idea is first to use the cascade control principle to model the under-damped system under PID control with a second-order system. Then, by making use of the sliding mode control outer loop, a robust, reduced-order response can be obtained to suppress the control overshoot. The proposed approach can also deal with time delay systems. Its $\mathrm{v}$ alidity is verified through simulation for some dynamic systems subject to hig hly nonlinear uncertainties, where overshoot remains an issue.
\end{abstract}

Keywords: sliding mode, cascade control, overshoot, robustness.

\section{Introduction}

Conventional PID c ontrol is quite popular in automatic control systems. The most important issue of a PID controller is th at its parameters need to be tuned properly. However, tuning a PID is not easy and in fact, many PID controllers in industry are not well-tuned.

There are some methods for tuning PID parameters. Based on knowledge of cha racterizing effects of each control parameter, engineers can adjust the P, I, and D gains until a desired response is obtained. However, this manual method is time-consuming and not always yields a desired response because changing one parameter may affect the performance designated by other two parameters. For over half a century the Ziegler an $d$ Nichols tuning methods [1] have been widely used in the context of aut o-tuning for PID controllers. In the first method, controller parameters are cal culated from an open-loop response of the process t o a step input (process reaction curve). In the second one, both I and D parameters are set to zero while $\mathrm{P}$ parameter is increased gradually until the system oscillates. The period of the oscillation (called ultimate period) and the P gain (called ultimate gain) are used to calculate the desired controller parameters. The Ziegler-Nichols rules can help the tuning process faster than the trial-and-error method.
However, they are not practical in many situations when experiments with open-loop or instable closed-loop can damage the process. To av oid this problem, many techniques such as relay feedback [2], approximate system identification [3], and cross-correlation [4] have been developed to estimate the ultimate gain and ultimate period in Ziegler-Nichols rules.

It is well-known that the control performance obtained by the Ziegler-Nichols tuning methods is just acceptable and the controller para meters need to be finetuned to provide the desired response [5]. While eliminating the steady error and shortening the settling time, the Ziegler-Nichols rules still result in a reasonable overshoot. This overshoot may be excessi ve and not acceptable in $\mathrm{m}$ any processes such as chemical or mechanical systems. In [6] Hang et al. proposed a method to reduce the control overshoot to $10 \%$ or $20 \%$, depending on applications, by using the set-point weighting. This may still appear inadequate for overshoot-sensitive systems.

When single-loop PID control systems cannot satisfy the control requirement, cascade PID control systems are often used. In [7], both optimization and auto-tuning methods are used for tuning cascade co ntrol systems. The results show that a cascade control s ystem gives better responses with shorter settling time and smaller overshoot compared with its single-loop control option.

In this paper, we propose to use the cascade control principle coupled with a sliding mode controller (SMC) at the outer loop to eliminate the overshoot of a st ep response of the PID-controlled inner loop. It is expected that not only overshoot is suppressed but such SMC prominent property as robustness to external disturbance, uncertainties and nonlinearities can also be achieved [8]. Using this method, the PID controller just needs to be tuned to obtain the desired settling time and steady-state error, while overshoot is not considered in the first stage. Based on the resulting closed-loop transfer function modeled by using the cascade control principle, a sliding mode controller (SMC) is then designed to co ntrol the input of the inner loop system in order to entirely suppress the control overshoot. Moreover, in the case the system is subject to an i nput time delay, which is identified as a cau se of deterioration of the overall 
control performance, the approach can al so be sh own effective if equipped with a suitable output predictor.

The remainder of the paper is organized as follows. After the introduction, Section 2 presen ts the modeling and sliding mode control design for cases wi th and without input time-delay. The approach is illustrated in Section 3 for a DC positioning system. Simulation results are given in Section 4 for the braking control system of a ski d-steering uninhabited ground vehicle. Finally, a conclusion is drawn in Section 5.

\section{Controller design}

\subsection{PID-controlled Process Modeling}

Figure 1 shows a conventio nal PID con troller in a closed-loop feedback system. Output of the controller is a function of the error between the PID loop reference, $r(t)$, and the output, $y(t)$, i.e. of $e_{0}=r-y$ :

$$
V=K_{P} e_{0}+K_{I} \int e_{0} d t+K_{D} \frac{d e_{0}}{d t} .
$$

Characteristics of a response to a unit step using this PID controller depend on the choice of its parameters. The proportional gain $\left(K_{P}\right)$ has the effect of reducing the rise time and it also reduces, but never eliminates, the steady-state error. The integral gain ( $\left.K_{I}\right)$ has the effect of annulling the steady-state error, but it may make the transient response worse. The derivative gain $\left(K_{D}\right)$ has the effect of increasing th e stability of the system, reducing the overshoot, and im proving the transient performance. To o btain a desired response, PID parameters need $\mathrm{t} o$ be tuned properly. By manually tuning or auto-tuning methods, the desired setting time and steady-state error can be obtained. Nevertheless, in some systems, no matter how well the PID parameters are tuned, overshoot of the step response still exists.

By making use of cascade control, whereby the output of the outer-loop controller is used to manipulate the setpoint of the inner-loop controller [7,9], and the symmetrical optimum principle [10], one can al ways model a PID-controlled process with overshoot into an equivalent second-order function:

$$
G(s)=\frac{Y(s)}{R(s)}=\frac{\omega_{n}^{2}}{s^{2}+2 \delta \omega_{n} s+\omega_{n}^{2}},
$$

where $\delta$ is the damping ratio and $\omega_{n}$ is the natural frequency. The system natural frequency and damping ratio can be calculated from the percentage of overshoot and peak time respectively as [5]:

$$
\omega_{n}=\frac{\pi}{t_{P} \sqrt{1-\delta^{2}}}, \delta=-\frac{\ln \left(M_{P}\right)}{\sqrt{\pi^{2}+\ln ^{2}\left(M_{P}\right)}},
$$

where $M_{P}$ and $t_{P}$ are percentage of overshoot and peak time, respectively. If the system is subject to an input time delay $\left(t_{d}\right)$ due to, e.g., h ydraulic actuation or sensing data transmission, the model (2) can be rewritten to incorposate $t_{d}$ as following

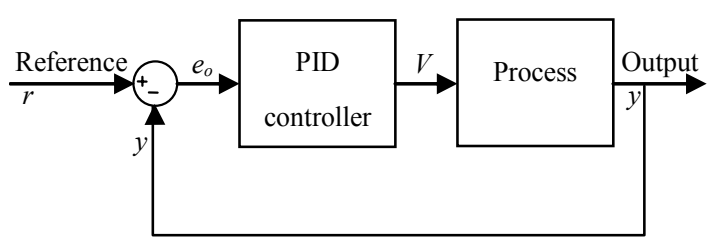

Figure 1. Closed-loop PID controller.

$$
G(s)=\frac{Y(s)}{R(s)}=\frac{\omega_{n}^{2}}{s^{2}+2 \delta \omega_{n} s+\omega_{n}^{2}} e^{-t_{d} s} .
$$

It is noted that the above sy stem may suffer from uncertainties such as nonlinear, modeling error and external disturbance. To deal with these uncertainties as well as overs hoot and delay issues, a sli ding mode controller (SMC) loop will be proposed in cascade with the PID-controlled system.

\subsection{Sliding Mode Controller Design}

Figure 2 shows the overal 1 control system, where the input of the PID contr oller is reg ulated by the output of the SMC. In this figure, $v$ is a n unknown input accounting for ex ternal disturbance, modeling error and parametric uncertainties.

Let the control system error be defined as

$$
e=y_{\text {ref }}-y \text {, }
$$

where $y_{\text {ref }}$ is the system reference or desired output. With the sliding function chosen as $S=\dot{e}+\lambda e$, where $\lambda$ is a positi ve scalar to be selected, let a Lyapunov function be $V_{L}=\frac{1}{2} S^{2}$. Taking its first time derivative yields $\dot{V}_{L}=S \dot{S}$, where

$$
\dot{S}=\ddot{e}+\lambda \dot{e}=\left(\ddot{y}_{\text {ref }}-\ddot{y}\right)+\lambda \dot{e} .
$$

Equation (2) gives

$$
\ddot{y}+2 \delta \omega_{n} \dot{y}+\omega_{n}^{2} y=\omega_{n}^{2} u .
$$

Substitution from (7) into (6) gives

$$
\begin{aligned}
& \dot{S}= \ddot{y}_{r e f}+2 \delta \omega_{n} \dot{y}+\omega_{n}^{2} y-\omega_{n}^{2} u+\lambda \dot{e} \\
&= \ddot{y}_{r e f}+2 \delta \omega_{n} \dot{y}_{r e f}-2 \delta \omega_{n}\left(\dot{y}_{r e f}-\dot{y}\right)+\omega_{n}^{2} y_{r e f} \\
&-\omega_{n}^{2}\left(y_{r e f}-y\right)-\omega_{n}^{2} u+\lambda \dot{e} \\
&= \ddot{y}_{r e f}+2 \delta \omega_{n} \dot{y}_{r e f}+\omega_{n}^{2} y_{r e f}-\left(2 \delta \omega_{n}-\lambda\right) \dot{e} \\
&-\omega_{n}^{2} e-\omega_{n}^{2} u . \\
& \text { or } \quad \dot{S}=\omega_{n}^{2} \varphi_{r e f}-\left(2 \delta \omega_{n}-\lambda\right) \dot{e}-\omega_{n}^{2} e-\omega_{n}^{2} u,
\end{aligned}
$$

where

$$
\varphi_{\text {ref }}=\frac{\left(\ddot{y}_{r e f}+2 \delta \omega_{n} \dot{y}_{r e f}+\omega_{n}^{2} y_{r e f}\right)}{\omega_{n}^{2}} .
$$

The equivalent control, $u_{e q}$, is obta ined at the nominal regime $(v=0)$ from condition $\dot{S}=0$ : 


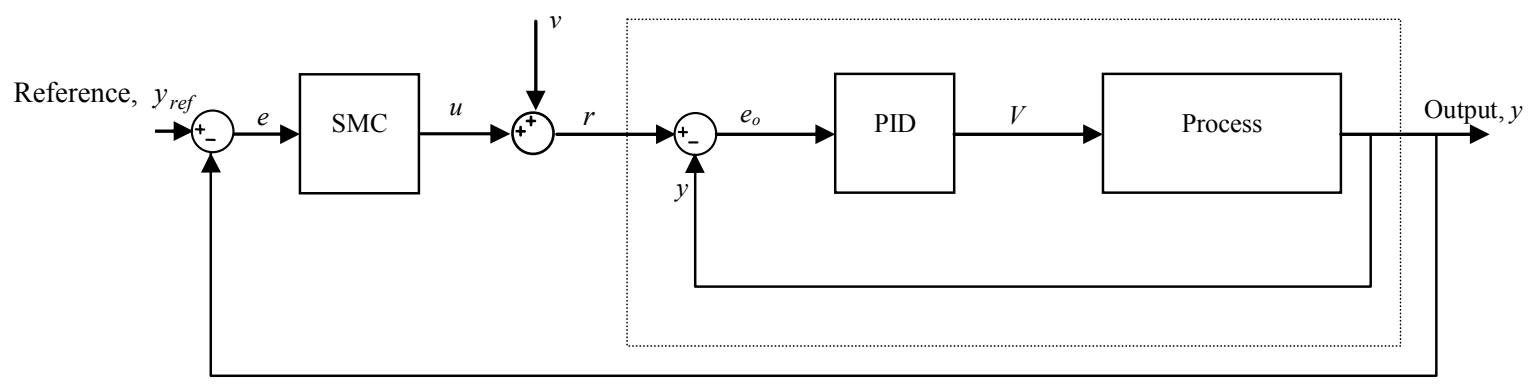

Figure 2. Cascade Sliding Mode - PID control

$$
u_{e q}=\varphi_{r e f}-\frac{\left(2 \delta \omega_{n}-\lambda\right)}{\omega_{n}^{2}-e .}
$$

Now for $v \neq 0$ the control law for SMC has the form of [11]:

$$
u=u_{e q}+u_{R} .
$$

Assuming $v$ is upper-bounded, $v \leq \rho$, one ca $\mathrm{n}$ easily verify that if the robust contrbill, $u_{R}$, is chosen as

$$
u_{R}=\operatorname{\rho sign}(S)
$$

then the reaching condition $\dot{V}_{L} \leq 0$ is satisfied since

$$
\begin{aligned}
\dot{V}_{L} & =S \dot{S} \\
& =S\left[\omega_{n}^{2} \varphi_{r e f}-\left(2 \delta \omega_{n}-\lambda\right) \dot{e}-\omega_{n}^{2} e-\omega_{n}^{2}\left(u_{e q}+u_{R}+v\right)\right] \\
& =-S\left[\omega_{n}^{2}\left(u_{R}+v\right)\right] .
\end{aligned}
$$

The control output of the SMC by (10) is then

$$
u=\varphi_{r e f}-\frac{\left(2 \delta \omega_{n}-\lambda\right)}{\omega_{n}^{2}} \dot{e}-e+\rho \operatorname{sign}(S) .
$$

The signum function in (12) usually induces high frequency oscillations in the control o utput, or socalled chattering. One of the commonly-adopted techniques to reduce this effect is the incorporation of of a saturation function [8].

Remark 1: The proposed m ethod may be applied generally for any overshoot-sensitive systems provided that their PID -controlled inner-loop step responses are known.

Remark 2: With robustness of the SMC, the proposed cascade control may be able to tol erate modeling errors, as well as to deal with such problems as external disturbanc e, uncertainties and nonlinearities.

\subsection{Case with input time delay}

Taking into account a tim e delay $t_{d}$ as in transfer function (4), system (7) can be rewritten as

$$
\ddot{y}(t)+2 \delta \omega_{n} \dot{y}(t)+\omega_{n}^{2} y(t)=\omega_{n}^{2} u\left(t-t_{d}\right),
$$

where

$$
\ddot{y}\left(t+t_{d}\right)=f\left(t+t_{d}\right)+\omega_{n}^{2} u(t),
$$

and

$$
f\left(t+t_{d}\right)=-2 \delta \omega_{n} \dot{y}\left(t+t_{d}\right)-\omega_{n}^{2} y\left(t+t_{d}\right) .
$$

To proceed, let us consider the natural frequency and damping ratio in the following intervals,

$$
\omega_{n 1} \leq \omega_{n} \leq \omega_{n 2}, \delta_{1} \leq \delta \leq \delta_{2},
$$

whose nominal values can be taken on average as [12]:

$$
\hat{\omega}_{n}=\omega_{n 1} \omega_{n 2}, \hat{\delta}=\delta_{1} \delta_{2} .
$$

Then $f\left(t+t_{d}\right)$ in (15) can be approximated with

$$
\hat{f}\left(t+t_{d}\right)=-2 \hat{\delta} \hat{\omega}_{n} \dot{y}\left(t+t_{d}\right)-\hat{\omega}_{n}^{2} y\left(t+t_{d}\right),
$$

which is assumed to be bounded by

$$
\hat{f}-f_{1} \leq F,
$$

where the values in the right hand side of (18) can be estimated by using an output predictor [13] and

$$
F=2\left(\delta_{2} \omega_{n 2}-\hat{\delta} \hat{\omega}_{n}\right) \dot{y}\left(t+t_{d}\right)+\left(\omega_{n 2}^{2}-\hat{\omega}_{n}^{2}\right) y\left(t+t_{d}\right) \text {. }
$$

Following the same design procedure described above and chossing the reaching condition $\dot{V}_{L} \leq-\eta S$, where $\eta$ is a positive constant to be determined $[8], \mathrm{t}$ he equivalent control as in (9) can obtained as

$$
u_{e q}(t)={ }_{\hat{\omega}_{n}^{2}}^{1}\left(-\hat{f}\left(t+t_{d}\right)+\ddot{y}_{d}(t)-\lambda \dot{e}\right) .
$$

The robust control of the form (11) can also be derived with the disc ontinuous gain $\rho$ chosen large enough to counteract the effects of uncertainties [12]:

$$
\rho \geq \underset{\omega_{n 1}^{2}}{F+\eta}+\left(\begin{array}{r}
\hat{\omega}_{n}^{2} \\
\omega_{n+}^{2}
\end{array}\right) u_{e q} .
$$

\section{DC motor positioning system example}

The proposed approach is illustrated first with a benchmark DC motor positioning control for linear systems. For this, consi der a system modeled by the following transfer function [5]:

$$
\begin{aligned}
& X(s)=\quad K \\
& \underline{V(s)} s\left[\left(J_{m} s+h_{m}\right)\left(L_{s}+R\right)+K^{2}\right]^{\prime}
\end{aligned}
$$


where $V$ and $x$ are applied voltage and position of the motor's shaft. The system parameters are given in Table I below:

TABLE I

DC MOTOR POSITION CONTROL PARAMETERS

\begin{tabular}{ll}
\hline Parameter & Value \\
\hline \hline Motor inertia & $J_{m}=3.2284 \mathrm{E}-6 \mathrm{~kg} \cdot \mathrm{m}^{2} \mathrm{~s}^{-2}$ \\
Viscous friction coefficient & $b_{m}=3.5077 \mathrm{E}-6 \mathrm{Nms}$ \\
Induction & $L=2.75 \mathrm{E}-6 \mathrm{H}$ \\
Resistance & $\mathrm{R}=4 \Omega$ \\
Electromotive force constant & $K=0.0274 \mathrm{NmA}^{-1}$ \\
PID parameters & $K_{P}=17, K_{I}=200, K_{D}=0$ \\
\hline
\end{tabular}

From tuning the PID co ntroller parameters, the system can obtain a fast $\mathrm{s}$ tep input response with zero steady-state error, but overshoot is still rather large. The proposed approach is used to solve the problem.

The results are shown in Fig. 3. When PID is used, a step reference (set-point) at the input (Fig. 3a) creates an oscillated voltage as the motor input (Fig. 3b) and results in a large overshoot at the motor shaft (Fig. 3c). In contrast, the SMC forces the PID input (control output of SMC, u) (Fig. 3a) an d the motor input (Fig. 3b) to eliminate completely the ove rshoot while still keeping the desired settling time for the whole system (Fig. 3c).

\section{Hydraulic Braking System for a UGV}

We consider next the problem of skid steer ing of an unmanned ground vehicle (UGV). For this UVG, important variables needed t o be co ntrolled at the low level include the engine s peed, left and ri ght braking forces, linear velocity and turning rate of the vehicle. The default algorithm for these vehicle controlled variables is the PID. Desirable features such as sim ple, generalpurpose and model-free, make PID controllers suitable for the control of the ve hicle. However, there are $m$ any components of the vehicle that exhibit nonlinearities and time delays which lead to high overshoots in the responses when using c onventional PID controllers. Because most components are correlated, overshoots in one controlled variable can result in adverse responses in the performance of others. For example, overshoot of a braking response (left or ri ght) may cause the turning rate to deviate away from its desired value. On the other hand, a fast rise time is a lways an essential requirem ent for the control system for th ese variables. Therefore, reducing overshoots of PID responses is an im portant task to enhance the contro 1 performance of the $\mathrm{UGV}$ [14]. The braking system consists of a voltage to current amplifier to s upply for a li near actuator, the actuator comprising a DC servo motor and a ball-s crew system, and a hydraulic cylinder driven by the actuator.

The actuator can be described by the following equations,

$$
\begin{aligned}
T_{m} & =K_{a} K_{i} V, \\
\frac{X}{T_{m}} & =\frac{K_{m}}{s\left(J_{m} s+B_{m}\right)},
\end{aligned}
$$

a)
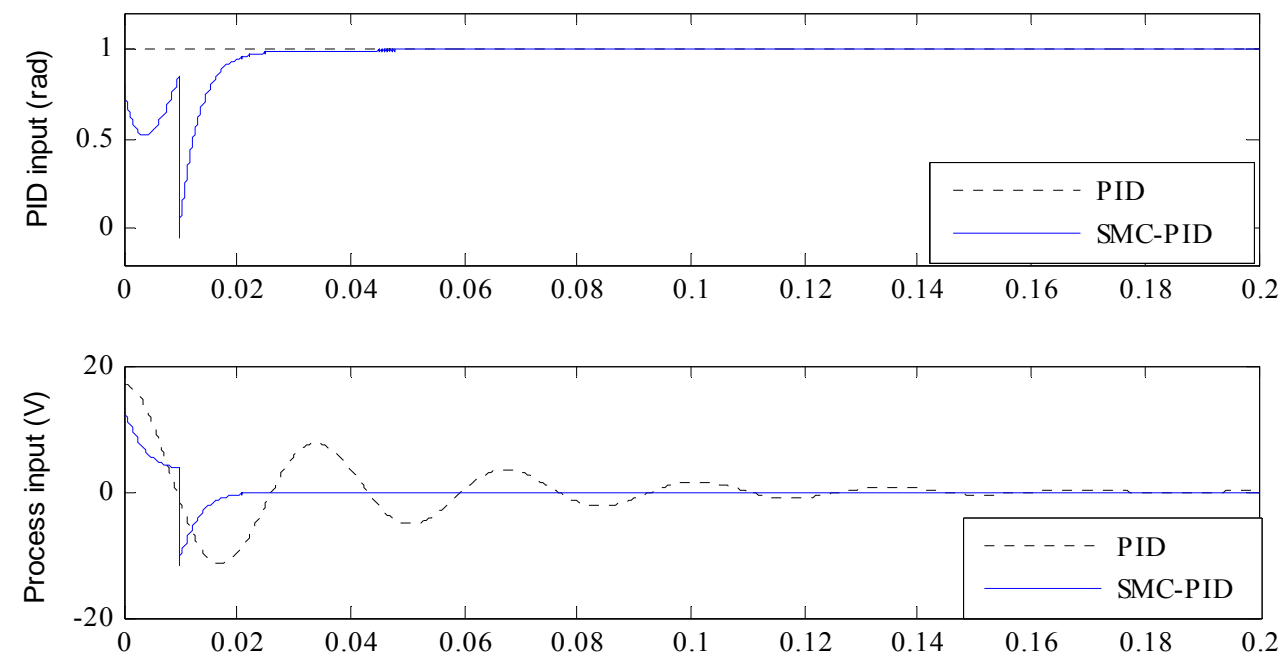

b)

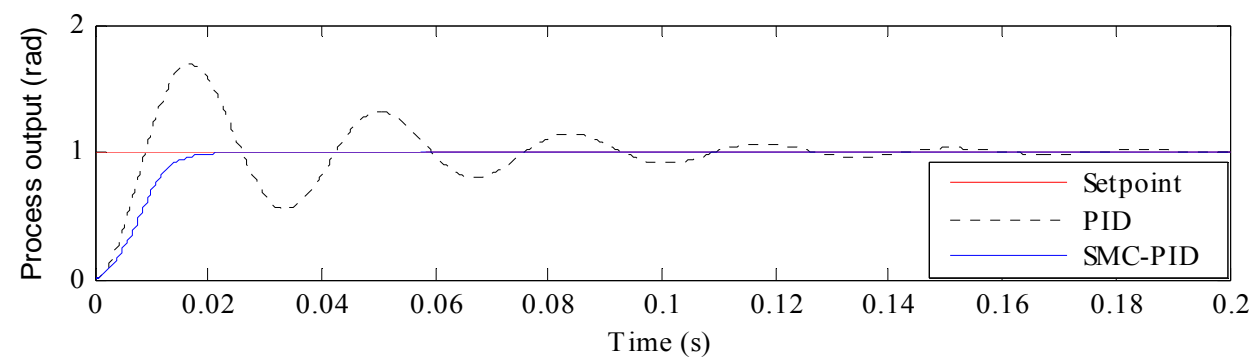

Figure 3. Response of PID controller (---) and SMC-PID (-) for DC motor position 
where $T_{m}, x$ and $V$ are the actuator's torque, position and the applie d voltage, $K_{a}, K_{i}$ are the voltage-current amplifier coefficient and motor torque coefficient, $J_{m}, B_{m}$ are the motor's moment of inertia and vis cous damping coefficient, and $K_{m}$ is the gear rati o inside the actuator. Values of the system parameters are provided in Table II. The complicated relationship between output $y$ and input $x$ of the hy draulic cylinder obtained from experimental data [15] by using the 1 east square identification method, as shown in Fig. 4, is estimated as

$$
y^{\prime}=f(x)=1.374 x^{2}-5.138 x+2.778 .
$$

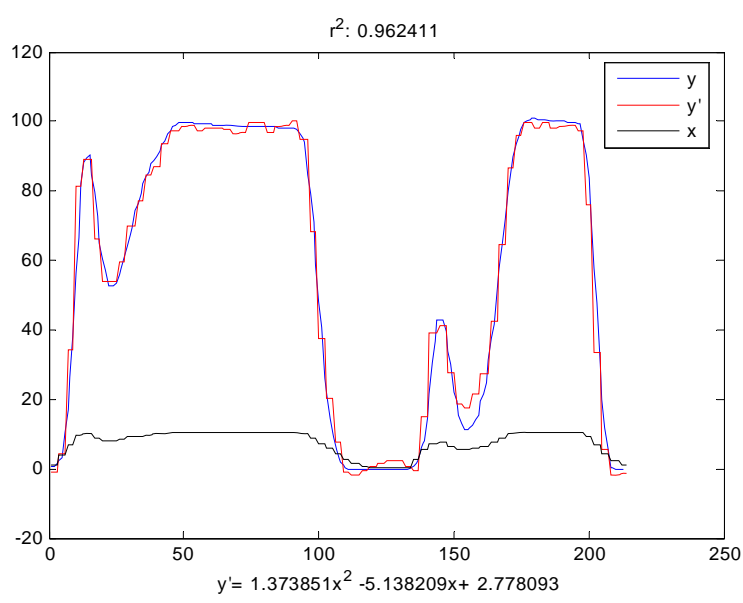

Figure 4. Pressure function approximation
TABLE II

BRAKING SYSTEM PARAMETERS

\begin{tabular}{ll}
\hline Parameter & Value \\
\hline \hline$K_{a}$ & $2 \mathrm{AV}^{-1}$ \\
\hline$K_{i}$ & $440 \mathrm{NA}^{-1}$ \\
\hline$K_{m}$ & 0.1815 \\
\hline$B_{m}$ & $33 \mathrm{Nms}$ \\
\hline$J_{m}$ & $0.3228 \mathrm{~kg} \cdot \mathrm{m}^{2} \mathrm{~s}^{-2}$ \\
\hline
\end{tabular}

For the UGV low-level contro 1, a pressure controller is designed with the assumption that the braking force is proportional to the pressure inside the hydraulic cylinder. A default PID-controller is used in the internal loop $f$ or hydraulic pressure control [15]. As overshoot appears to be a pr oblem no $\mathrm{m}$ atter how the PI D controller parameters are tuned, the proposed cascade PID-SMC is used to solve the problem.

The results are shown in Fi g. 5 for both the PID and SMC-PID controllers. From step refer ences, it is observed that the PID cas e alone possesses a large overshoot at the output (pre ssure) while the cascaded SMC can control the PID in put (control output of SMC, u) (Fig. 5a) to force the system output to a non-overshoot step response (Fig. 5c).

Fig. $6 \mathrm{~s}$ hows responses of the controlle rs with a disturbance representing a load change. The amplitude of disturbance is about $60 \%$ of maximum torque provided by the actuator (Fig. 6a). T he PID co ntroller cannot regulate the output bra king disc to the desired value while the SMC is st ill able to control the PID input (SMC output, $u$ ) (Fig. 6b) in a robust way to compensate

a)
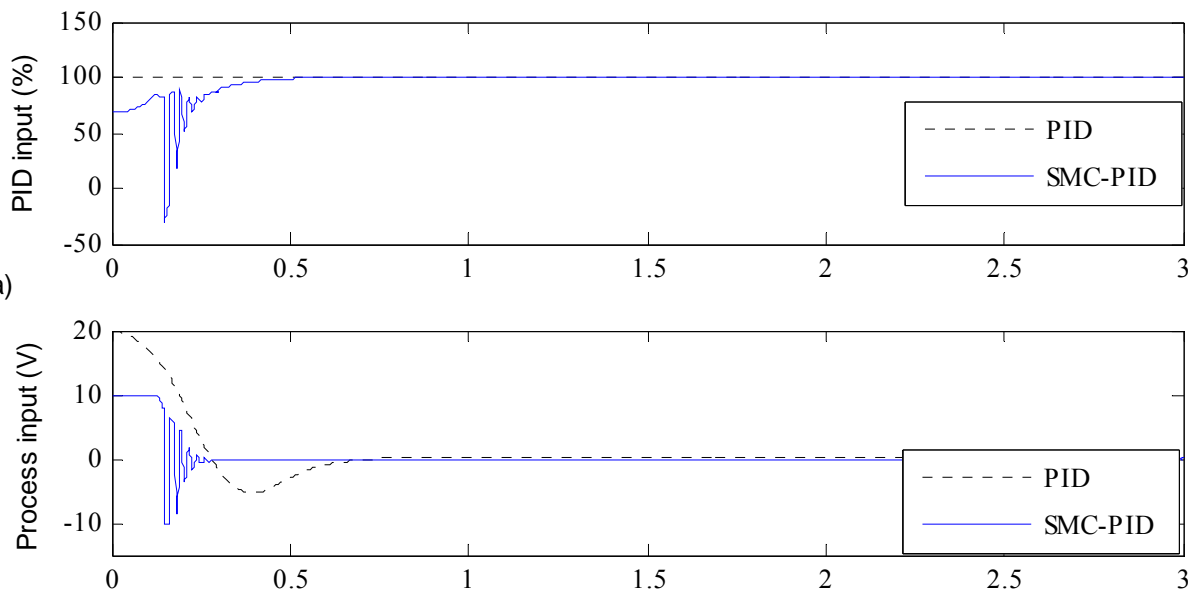

b)

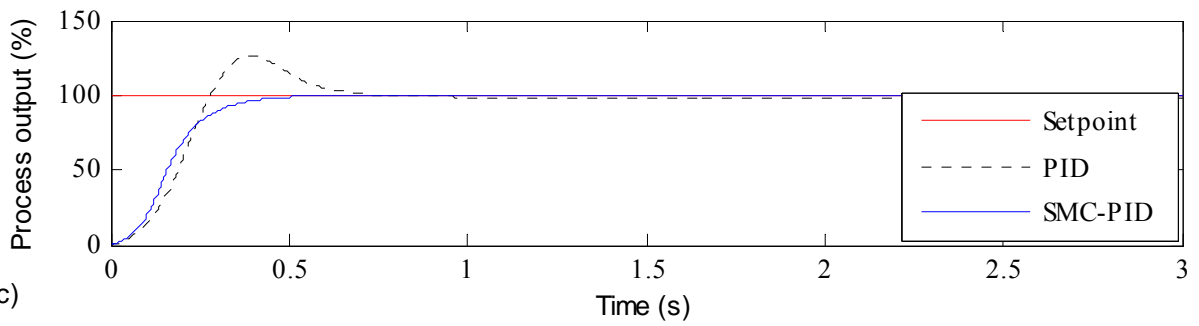

Figure 5. Responses of PID (---) and SMC-PID (-) control for UGV hydraulic braking system 


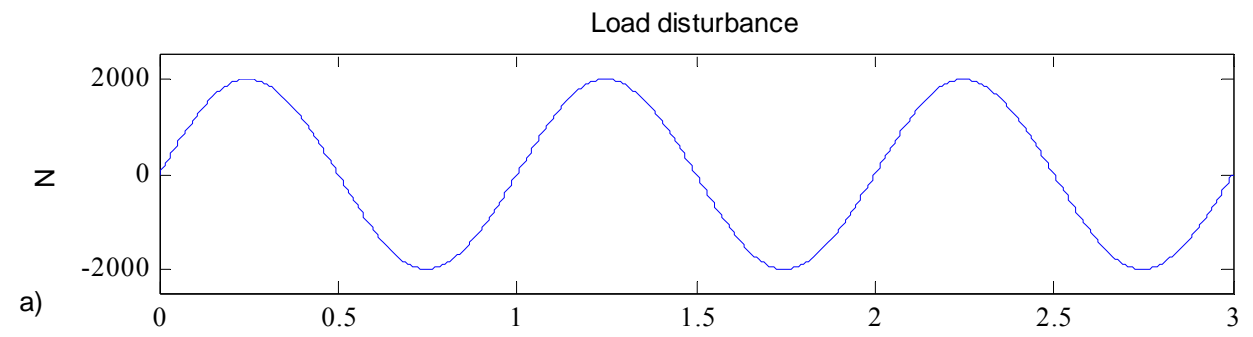

b)
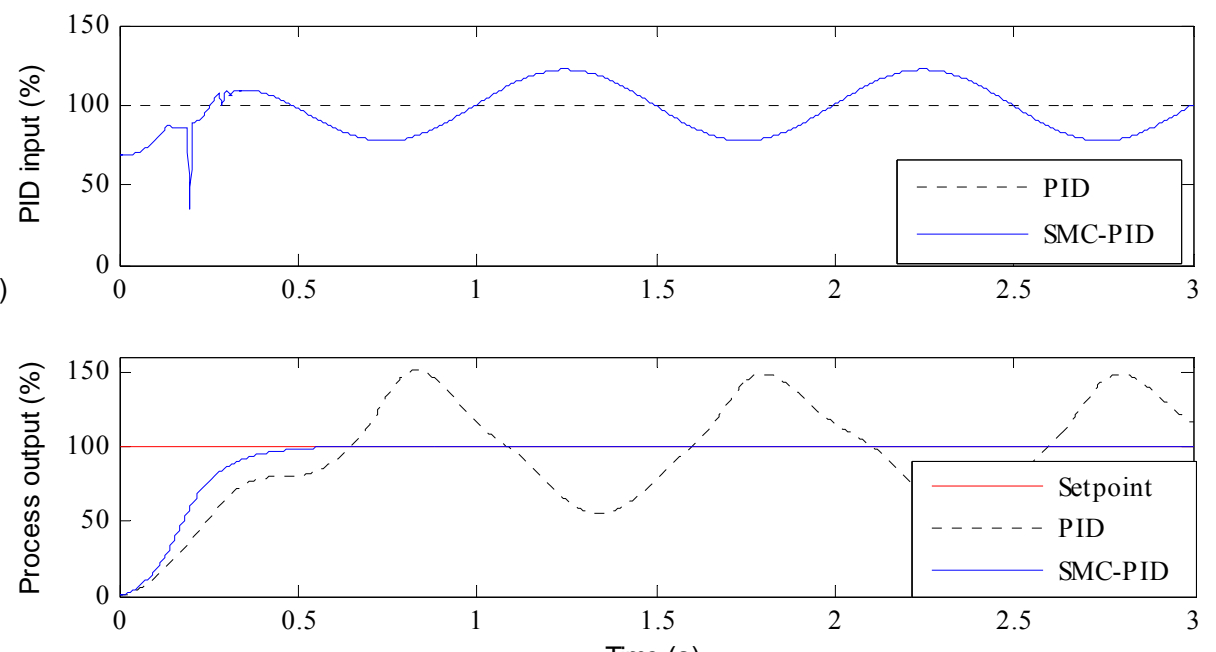

c)

Time (s)

Figure 6. Responses of PID (---) and SMC-PID (-) control with external disturbance
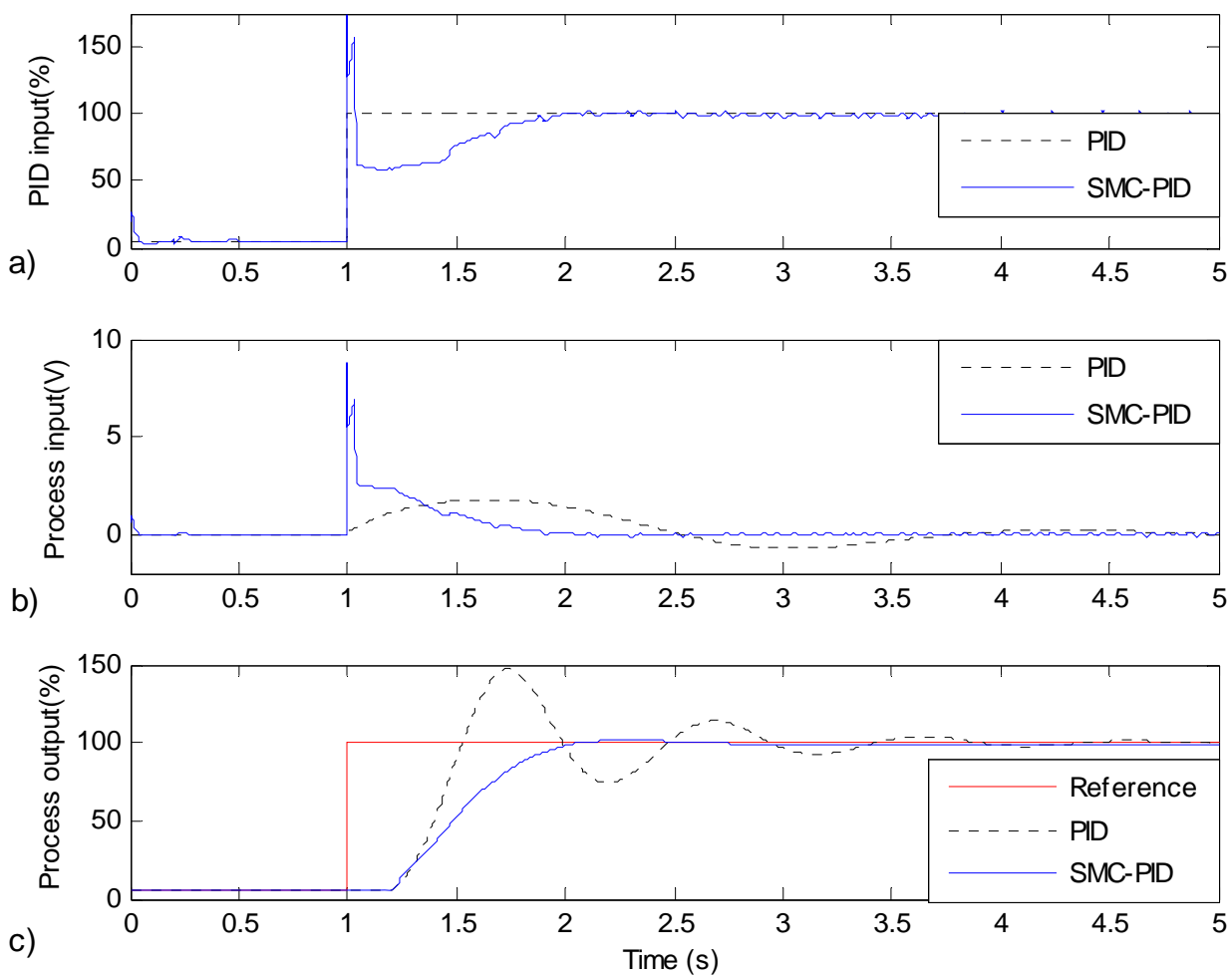

Figure 7. Responses of PID (---) and SMC-PID (-) control for UGV hydraulic braking system, in case of time-delay 
for the disturbance. As a result, the error of the SMCPID is found less than $0.6 \%$ compared with $50 \%$ of the PID (Fig. 6c). This is explained by the prominent feature of sliding mode control in producing robust, reducedorder time responses, and thus, suppressing successfully the step response control overshoot.

To illustrate the effectiveness of the proposed approach for tim e-delay systems, the ab ove braking system is cons idered again taking into account now a time-delay of around 0.2 second between the input and the output [11]. This time-delay leads to around $50 \%$ of overshoot if PID controller is used alone (Fig. 7c). With estimation owing to the i ncorporation of an ouput predictor, the designed SMC-PID controller can suppress the overshoot to a very small value (Fig. 7c ). Thus the results show that the proposed method can be effective for systems with an input time-delay.

\section{Conclusion}

We have presented a cascade sliding $\mathrm{m}$ ode-PID controller for robust, non-overshoot time responses. The proposed approach can be applied for any PID-controlled system if its step response is known. From an equivalent transfer function of the PID inner-loop system, a sliding mode controller is designed to force the input of the PID so that the control overshoo $t$ is fully e liminated. An interesting feature is the ap proach remains effective for time-delay systems. Its valid ity is verified through a benchmark DC positioning system and a UGV braking system used for its skid st eering. Simulation results for the UGV hydraulic braking sy stem indicate that the proposed method can succ essfully suppress control overshoots while preserving high quality of other performance criteria such as settling tim e and steadystate error. By using the proposed approach, the control system can also exhibit strong robustness against uncertainties such as external disturbance, nonlinearities as well as input time delay.

\section{Acknowledgement}

This work is supported by the Vietnam Ministry of Education and Training and by the ARC Centre of Excellence programme, funded b y the Australian Research Council (ARC) and the New South Wales State Government.

\section{References:}

[1] J. G. Ziegler and N. B . Nichols, "Optimum setting for automatic controllers," ASME Transactions, vol. 64, 759-768, (1942).

[2] C.-C. Yu, Autotuning of PID controllers: relay feedback approach. London; New York: Springer, (1999).

[3] C. C. Hang, T. H. Lee, and T. T. Tay, "The use of recur sive parameter estimation as an auto-tuning aid," Proc. ISA Annual Conf., 387-396, (1984).

[4] C. C. Hang and K. K. Sin, "On-line auto tuning o f PID controllers based on the cr oss-correlation technique," IEEE Transactions on Industrial Electronics, vol. 38, 428-437, (1991).

[5] G. F. Franklin, J. D. Powell, and A. Emami-Naeini, Feedback control of dynamic systems, 4th ed. Upper Saddle River, NJ: Prentice Hall, (2002).

[6] C. C. Hang, K. J. Astrom, and W. K. Ho, "Refinements of the Ziegler-Nichols tuning form ula," Control Theory and
Applications, IEE Proceedings D, vol. 138, pp. 111-118, (1991).

[7] M. Zhuang and D. P. Ather ton, "Optimum cascade PID controller design for SISO systems," Proc. IEE Conference on Control, Warwick UK, vol. 1, 606-611, (1994).

[8] J. Y. Hung, W. Gao, and J. C. Hung, "Variable structure control: a survey," IEEE Transactions on Industrial Electronics, vol. 40, 2-22, (1993).

[9] W. Tan, J. Liu, T. Chen, and H. J. Marquez, "Robust Analysis and PID Tuning of Cascade Co ntrol Systems," Chemical Engineering Communications, vol. 192, 1204-1220, (2005).

[10] A.A. Voda and I .D. Landau, "A method for the autocalibration of PID contr ollers," Automatica, Vol. 31, No. 1, pp. 41-53, (1995).

[11] Q.P. Ha, D.C. Rye, and H.F. Durrant-Whyte, " Robust sliding mode control with application, " International Journal of Control, Vol. 72, No. 12, 1087-1096, (1999).

[12] T. H. Tran, N. M. Kwok, M. T. Nguyen, Q. P. Ha, and G. Fang, "Sliding Mode-PID Controller for Robust L ow-level Control of a UGV," Proc. of the IEEE Conference on Automation Science and Engineering, 684-689, (2006).

[13] R. Young-Hoon and O. Jun-Ho, "Sliding mode control with uncertainty adaptation for uncertain input-delay systems," International Journal of Control, vol. 73, 1255- 260, (2000).

[14] T. H. Tran, Q. P. Ha, R. Grover, and S. Scheding, "Modelling of an a utonomous amphibious vehicle," Proc. of the 2004 Australian Conference on Robotics and Automation, (2004).

[15] Q. P. Ha, T. H. Tran, S. Scheding, G. Dissanayake, and H. F. Durrant-Whyte, "Control Issues of an Autono mous Vehicle," Proc. the 22th International Symposium on Automation and robotics in Construction, (2005).

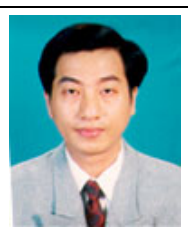

Thanh Hung Tran

Faculty of Engineering,

University of Technology Sydney (UTS)

PO Box 123, Broadway NSW 2007,

Australia

T.H. Tran is currently a PhD. stude nt at the Faculty of Engineering, UTS. He obtained his MEng in the field of telecommunication in 2000 from Ho Chi M inh City University of T echnology, Vietnam. His research interests include r obotics, control technologies, and vehicleterrain interaction

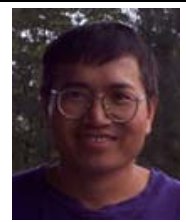
Quang Ha
Faculty of Engineering,
University of Technology Sydney (UTS)
PO Box 123, Broadway NSW 2007
Australia

Q. P. Ha is cur rently an Associate Professor at the Faculty of Engineering, UTS. He obtained his $\mathrm{PhD}$ in Electrical Engineering in 1993 from Moscow Power Engineering Institute, Russia. His research interests include $\mathrm{r}$ obust control and esti mation, robotics, and computational intelligence applications. He is a member of the IEEE.

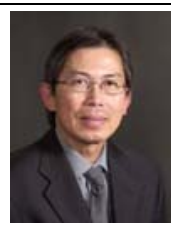

\section{Hung Nguyen}

Faculty of Engineering,

University of Technology Sydney (UTS)

PO Box 123, Broadway NSW 2007,

Australia

H. T. Nguyen is cur rently Professor of E lectrical Engineering, Associate Dean (Research) at the Fa culty of engineering, UTS, and a Member of the Order of Australia (AM). He is Director of the Key Centre for Health Technologies at UTS. He received his PhD in 1980 from the University of Newcastle, Australia. His research interests are in biomedical engineering, advanced control and instrumentation, and artificial intelligence. He is a senior member of the IEEE. 